\title{
BIORREATORES COM MEMBRANAS: UMA ALTERNATIVA PARA O TRATAMENTO DE EFLUENTES
}

\author{
I. R. CADORE ${ }^{1}$, M. K. da SILVA ${ }^{1}$, L. D. POLLO ${ }^{1}$, I. C. TESSARO ${ }^{1}$ \\ ${ }^{1}$ Universidade Federal do Rio Grande do Sul, Departamento de Engenharia Química \\ E-mail para contato: igorcadore@hotmail.com.br
}

RESUMO - A associação da tecnologia de separação por membranas aos processos de lodos ativados representa uma importante evolução nos processos de tratamento de efluentes, apresentando vantagens como menor espaço de instalação, alta qualidade do efluente tratado e baixa produção de lodo durante o processo. Este trabalho tem como objetivo obter uma melhora de desempenho com relação às condições de aeração, a partir da modificação de algumas variáveis do sistema de Biorreatores com Membranas Submersas (SMBR). As membranas utilizadas foram fibras ocas de poli(éter-imida) (PEI). Diferentes condições de aeração foram testadas e, observou-se que, para todas as condições de aeração, houve decréscimo contínuo e acentuado do fluxo permeado para $70 \%$ do fluxo inicial em 16 horas de experimento. A partir de um reescalonamento do sistema, aumentando o volume do biorreator e diminuindo a densidade de empacotamento do módulo, observou-se uma menor queda do fluxo permeado, atingindo valores em torno de $80 \%$ do fluxo permeado inicial após o mesmo tempo de operação. Estes resultados demonstram que o tamanho do biorreator e a densidade de empacotamento devem ser cuidadosamente projetados para aumentar o tempo de operação do sistema, e desta forma, diminuir o consumo energético.

\section{INTRODUÇÃO}

Em busca da preservação das fontes hídricas naturais, o reuso das águas de processo tem sido amplamente difundido. Diante desta necessidade, surgem como alternativa os Biorreatores com Membranas Submersas (SMBR), que consistem em um processo que associa o tratamento biológico utilizado nos processos de lodos ativados à tecnologia de separação por membranas.

Nos SMBR, a separação é realizada pela filtração com membranas e não por sedimentação, como nos processos convencionais, garantindo um efluente de alta qualidade, uma vez que a biomassa é quase totalmente retida pela membrana, devido à diferença de tamanho entre os poros da membrana e o material a ser retido. Todavia, os SMBR possuem uma desvantagem em relação ao tratamento convencional: uma taxa de consumo energética elevada devido à incrustação da superfície da membrana (em inglês, fouling), acarretando em perda de capacidade de tratamento e necessidade de limpezas frequentes das membranas.

Dentre as principais condições operacionais de um sistema SMBR que afetam o fouling insere-se a pressão transmembrana (PTM), a velocidade tangencial, a vazão de aeração, o tempo 


\section{9 a 22 de outubro de 2014 \\ Florianópolis/SC}

de retenção hidráulica, a idade do lodo e a frequência de permeação/retrolavagem (Chang et al.,2002 e Liao et al.,2004). Do ponto de vista da questão energética, atenta-se à aeração, que é responsável por 80-90\% dos custos totais de operação de um SMBR (Melin et al., 2006).

Segundo Lebegue et al. (2008), a homogeneidade das condições hidrodinâmicas é um fator importante nos SMBR e está relacionada à qualidade do projeto do sistema de aeração. A densidade de empacotamento do módulo (relação entre a área de permeação e o volume do módulo) também exerce influência na homogeneidade de aeração no sistema SMBR. Em densidades de empacotamento elevadas, algumas fibras podem se fundir através das tortas formadas na superfície (Yeo e Fane, 2005), podendo ocorrer a formação de zonas estagnadas no centro de feixes de fibra oca (Lebegue et al., 2008).

A aeração utilizada em sistemas SMBR têm três funções principais: fornecer oxigênio aos microrganismos, manter o lodo ativado em suspensão e limitar o fouling pela remoção das partículas aderidas à superfície. Por estas razões, a vazão de aeração é o principal parâmetro para o controle do fouling. Diversos trabalhos na literatura (Ueda et al., 1997; Chua et al., 2002; Delgado et al., 2008) demonstram que a tendência ao fouling foi reduzida devido a um aumento na velocidade superficial de gás, atingindo um valor limite de aeração benéfico ao sistema.

A existência deste limite de aeração significa que podem ocorrer efeitos adversos, como por exemplo, apresentado no trabalho de Meng et al. (2008), onde a aeração intensa resultou na quebra dos flocos e promoveu a liberação de partículas coloidais e solúveis, agravando o fouling. Em níveis elevados de aeração, as partículas tendem a ser menores, aumentando a chance de formação do fouling no interior dos poros devido à maior presença de partículas solúveis e coloidais (Wu et al., 2012).

Pela investigação dos efeitos globais da aeração, a tendência é o surgimento de um valor limite para a eficiência de filtração. Além disso, devido às diversas configurações de SMBR e condições operacionais utilizadas, não é possível fixar um valor ótimo de taxa de aeração. Com base nestes estudos, o objetivo deste trabalho é buscar um melhor desempenho do sistema SMBR estudado a partir da modificação da densidade de empacotamento do módulo, da área de seção transversal do tanque e da vazão de aeração.

\section{MATERIAIS E MÉTODOS}

\subsection{Sistema de Biorreator com Membranas}

O sistema SMBR foi desenvolvido de forma a permitir o estudo das condições operacionais do processo e manter as necessidades essenciais do lodo, bem como realizar testes de longa duração de forma automatizada e a aquisição de dados de forma contínua (on-line). A Figura 1 apresenta um fluxograma simplificado do sistema. Mais detalhes sobre o sistema podem ser encontrados no trabalho de Silva (2009). 


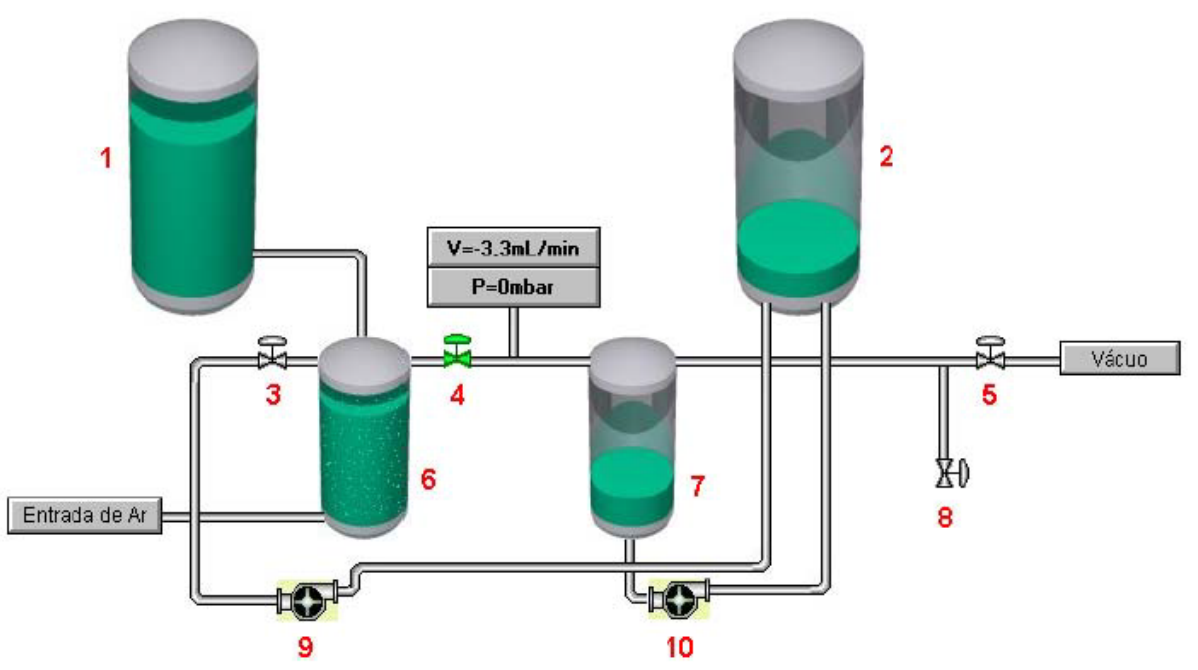

Figura 1 - Ilustração do sistema SMBR: 1 - Tanque de armazenamento do efluente; 2 - Tanque de armazenamento do permeado; 6 - Biorreator; 7 - Tanque de coleta do permeado; 3, 4, 5 e 8 Válvulas de controle do tipo solenoide; 9 - Bomba de retrolavagem; 10 - Bomba de esvaziamento do tanque 7. Adaptado de Silva, 2009.

Primeiramente, utilizou-se um biorreator de 4,5 L e um medidor de vazão de engrenagens ovais, na linha entre o tanque 6 e o tanque 7 (SMBR 1). Posteriormente, aumentou-se o volume do biorreator para $8 \mathrm{~L}$ e fez-se uso de uma célula de carga, sendo esta fixada ao tanque 7 suspenso, desta forma, a vazão é medida pela diferença de massa ao longo do tempo (SMBR 2).

Membranas: Nos experimentos foram utilizadas membranas do tipo fibras ocas de poli(éter-imida) com diâmetro médio de poros na superfície externa da membrana entre 0,1 e 0,5 $\mu \mathrm{m}$. As membranas foram fornecidas pela PAM - Membranas Seletivas Ltda em fibras de $45-$ $50 \mathrm{~cm}$ de comprimento. $\mathrm{O}$ diâmetro externo das fibras está entre $0,8-1,0 \mathrm{~mm}$; a permeância hidráulica informada pelo fabricante é de $300 \mathrm{~L} /\left(\mathrm{m}^{2} . h . b a r\right)$, porém com os testes de caracterização observa-se que este valor se encontra na faixa de $60-150 \mathrm{~L} /\left(\mathrm{m}^{2} . h . b a r\right)$; isto se deve provavelmente, a menor área de membrana utilizada. A Figura 2 ilustra um módulo de membranas construído com 53 fibras, $24 \mathrm{~cm}$ de comprimento, totalizando uma área de 0,036 m².

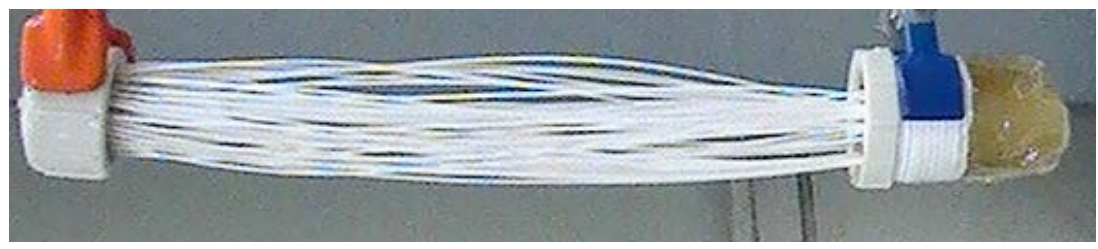

Figura 2 - Módulo de membranas utilizado no sistema SMBR: 53 fibras com $24 \mathrm{~cm}$ de comprimento, densidade de empacotamento de $296 \mathrm{~m}^{2} / \mathrm{m}^{3}$ e área útil de membrana de $0,036 \mathrm{~m}^{2}$. 
Sistema de aeração: O sistema é composto basicamente de um aerador, um rotâmetro e um compressor. O faixa de medição do rotâmetro é de 0 a $10 \mathrm{~L} / \mathrm{min}$, na pressão de 2 bar. O aerador foi construído utilizando 6 pedras porosas de aquário, dispostas simetricamente em torno de um distribuidor de ar, podendo ser visualizado na Figura 3.
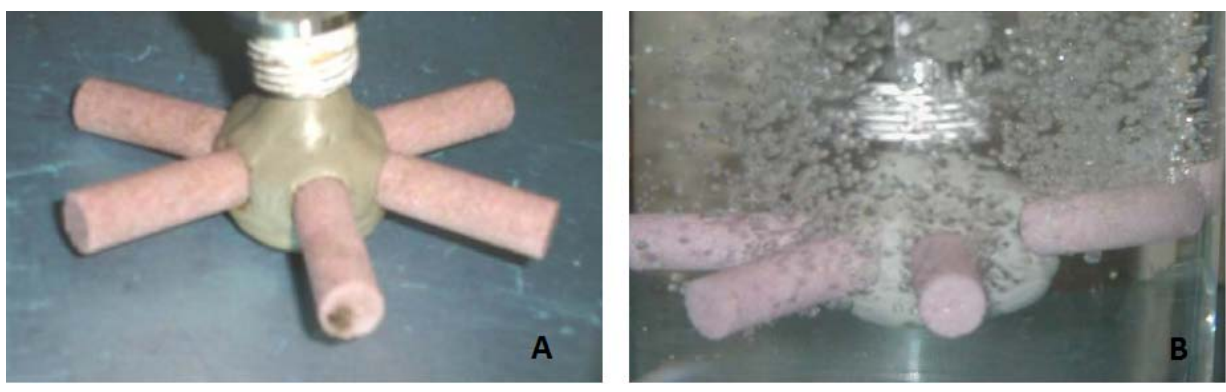

Figura 3 - Aerador utilizado no sistema MBR: A) Aerador; B) Formação de bolhas. Adaptado de Silva, 2009.

\subsection{Caracterização da Membrana}

Permeância Hidráulica: A permeância hidráulica é determinada a partir de medidas de fluxo permeado obtido em cada PTM, podendo variar de 100 a 700 mbar. A partir da curva fluxo permeado (Jp) versus diferença de pressão $\left(\Delta \mathrm{P}=\mathrm{P}_{\text {atm }}\right.$ - $\left.\mathrm{P}_{\text {vácuo }}\right)$ obtida destes pontos experimentais pode-se determinar a permeância hidráulica (Lp) a partir da Equação 1:

$$
J p=L p \times \Delta P
$$

\subsection{Procedimento experimental}

Compactação da membrana: A compactação da membrana consiste no adensamento da sua microestrutura quando aplicada determinada diferença de pressão. A pressão de 300 mbar foi utilizada para compactação da membrana, até que fosse atingido um fluxo permeado de água estável. Esse procedimento foi realizado antes de cada experimento, para permitir a análise dos fenômenos de compactação e de fouling separadamente.

Determinação da condição ótima de aeração: Para determinar a condição ótima de aeração, dois sistemas SMBR distintos foram estudados, cujas condições operacionais podem ser visualizadas na Tabela 1. 
Tabela 1 - Condições operacionais dos sistemas SMBR estudados.

\begin{tabular}{|c|c|c|}
\hline Sistema SMBR & SMBR 1 & SMBR 2 \\
\hline Volume Biorreator (L) & 4,5 & 8 \\
\hline Diâmetro Biorreator (cm) & 11 & 15 \\
\hline Vazão de aeração (L/min) & $2,4,6$ e 8 & 2,5 e 8 \\
\hline Vel. Superficial (m/h) & 11,$6 ;$ 23,1; 34,7 e 46,2 & 6,$8 ; 16,9$ e 27,1 \\
\hline Medida de vazão & Medidor de engrenagens ovais & Célula de carga \\
\hline Diâmetro do módulo (cm) & 1,27 (1/2 in) & 1,91 (3/4 in) \\
\hline Pressão de operação (mbar) & 600 & 400 \\
\hline
\end{tabular}

\section{RESULTADOS E DISCUSSÃO}

\subsection{Experimentos no SMBR1}

Os resultados dos experimentos realizados antes da modificação do biorreator (SMBR1) estão apresentados na Figura 4. O fluxo inicial para os 4 testes foram bem próximos, em torno de 22,5 L/(m².h). A membrana utilizada apresentou permeância hidráulica em torno de 152 $\mathrm{L} /\left(\mathrm{m}^{2}\right.$.h.bar). Os resultados estão apresentados em termos de fluxo normalizado, que consiste na razão entre o fluxo em um determinado tempo e o fluxo inicial.

O melhor desempenho do sistema foi obtido na vazão de $6 \mathrm{~L} / \mathrm{min}$ (no gráfico, 34,7 m/h), resultando em um fluxo final igual a 70\% do fluxo inicial, após 16 horas de operação. Pelo gráfico, observa-se que o nível mais alto de aeração $(8 \mathrm{~L} / \mathrm{min}$, ou 46,2 m/h) provocou uma queda no desempenho do sistema, podendo ter ocasionado a quebra dos flocos e fouling mais acentuado, como relatado no trabalho de Meng et al. (2008), atingindo um fluxo final de 50\% do fluxo inicial, abaixo dos 65-70\% obtido pelos níveis menores de aeração. Os 3 menores níveis de aeração apresentaram resultados semelhantes, podendo ser um indicativo de que a homogeneidade no interior do feixe não foi alcançada. 


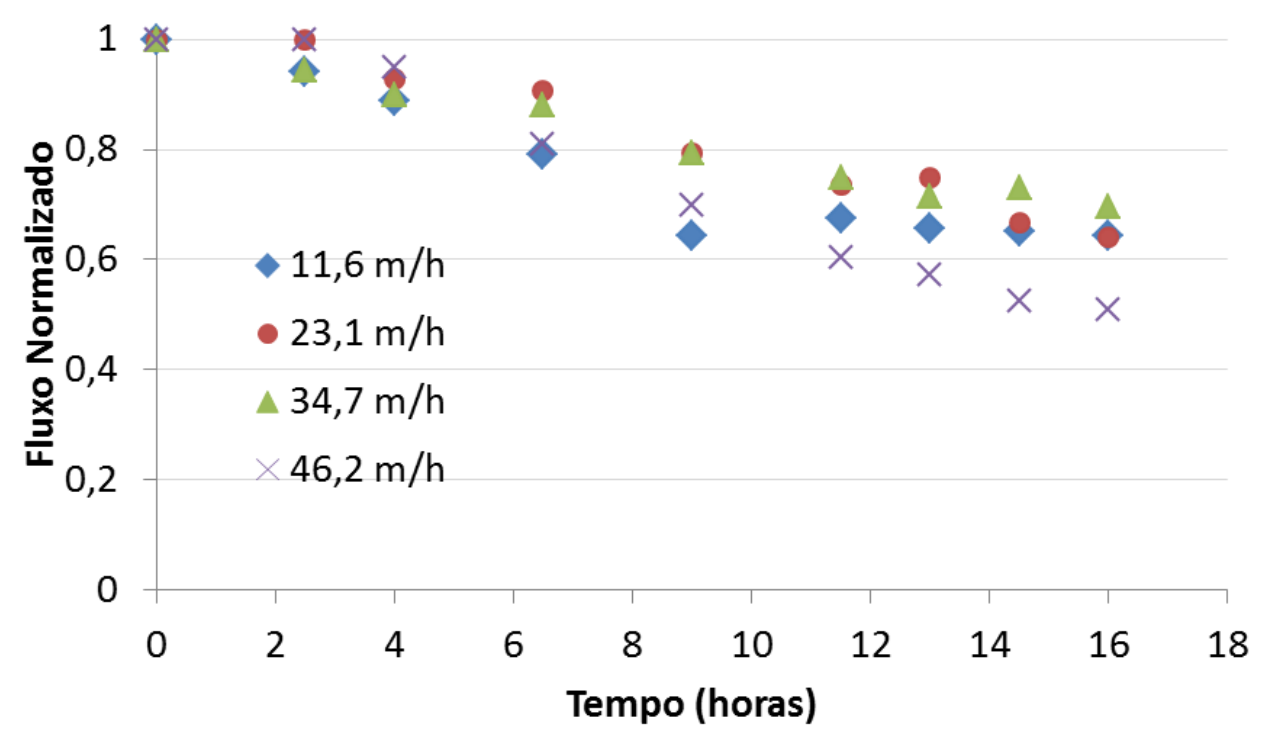

Figura 4 - Fluxo permeado normalizado versus tempo para diferentes intensidades de aeração (SMBR 1).

Vale ressaltar que, nesta primeira batelada de experimentos a vazão foi medida em um medidor de vazão de engrenagens ovais, e, ao longo do experimento, o escoamento de permeado pode tornar-se bifásico (ar e água), prejudicando a precisão das medidas. Medidores de vazão deste tipo comumente apresentam problemas se o escoamento for bifásico e as fases não forem separadas para a medida de vazão (Oliveira, 2007).

\subsection{Experimentos no SMBR 2}

A Figura 5 apresenta os resultados para os testes realizados com um biorreator de maior volume, denominado SMBR 2. O fluxo inicial para os 3 testes foi em torno de $37 \mathrm{~L} /\left(\mathrm{m}^{2} . \mathrm{h}\right)$. A permeância hidráulica da membrana utilizada foi de $65 \mathrm{~L} /\left(\mathrm{m}^{2}\right.$.h.bar).

Todos os níveis de aeração testados apresentaram resultados distintos, de maneira que a menor densidade de empacotamento do módulo resultou em uma maior homogeneidade de aeração no interior do feixe. Outro fator que contribuiu para verificar as diferenças de fluxo permeado para os diferentes níveis de aeração foi a troca do medidor de vazão para uma célula de carga, diminuindo os erros de medida ocorridos nos testes do SMBR 1.

O melhor valor de fluxo final foi obtido em $6,8 \mathrm{~m} / \mathrm{h}$ (2 L/min), a menor vazão de aeração testada. Assim como ocorreu no sistema SMBR 1, em vazões mais elevadas houve uma diminuição do fluxo normalizado, corroborando com resultados obtidos por Meng et al. (2008) e indicando a ocorrência de um limite máximo de vazão de aeração. 


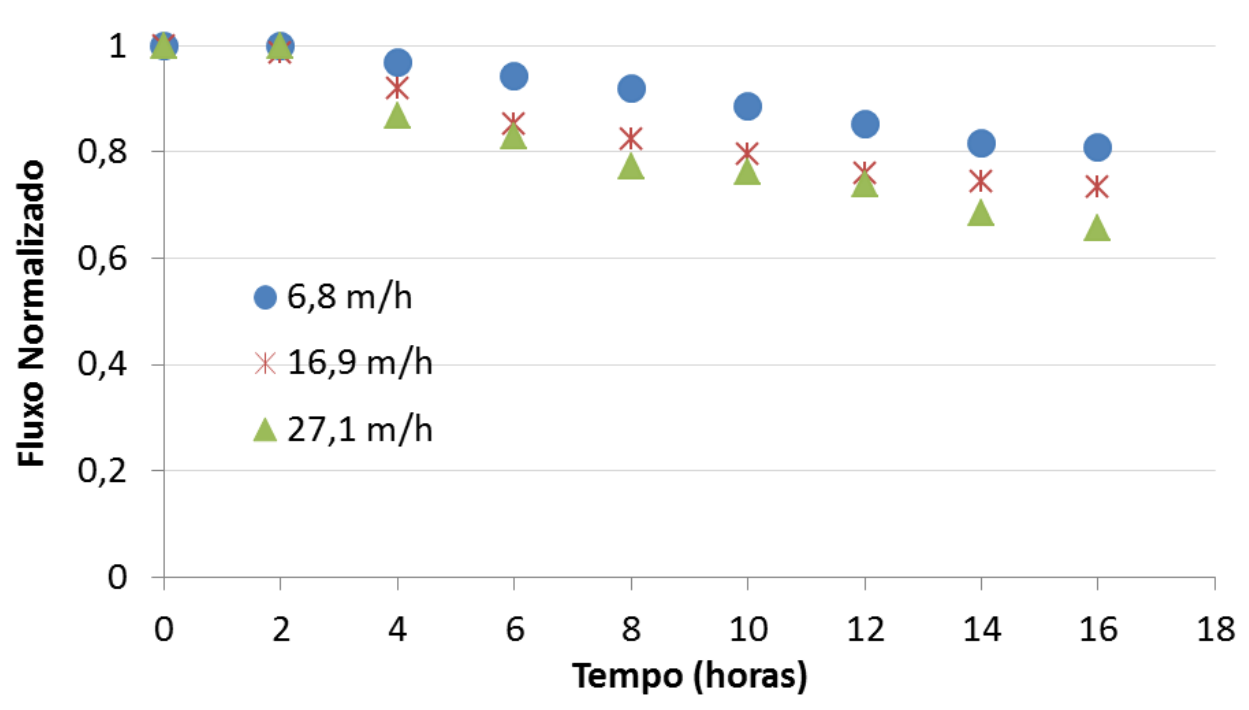

Figura 5 - Fluxo permeado normalizado versus tempo para diferentes intensidades de aeração (SMBR 2).

\section{CONCLUSÕES}

Os resultados obtidos neste trabalho mostraram que o melhor fluxo final para os testes de SMBR 1 foi de $70 \%$ a uma taxa de aeração correspondente a $6 \mathrm{~L} / \mathrm{min}$. Nos testes de SMBR 2, o melhor fluxo final foi de $80 \%$ a uma taxa de aeração de $2 \mathrm{~L} / \mathrm{min}$, mesmo com valores de permeância hidráulica inferiores. Foi possível confirmar a existência da vazão limite de aeração benéfica ao fouling e que esta depende de cada sistema em estudo. Além disso, observou-se que as medidas de vazão realizadas com a célula de carga apresentaram maior precisão, e, desta forma, é possível visualizar melhor as diferenças de fluxo nos testes do SMBR 2.

A partir dos resultados obtidos, conclui-se que o sistema SMBR estudado apresentou um melhor desempenho após a implementação das modificações propostas. Este resultado demonstra a importância do projeto, indicando que o tamanho do biorreator, a densidade de empacotamento das membranas e a taxa de aeração são parâmetros essenciais para diminuiu e/ou evitar os efeitos adversos ligados a estes processos como polarização por concentração e fouling.

\section{REFERÊNCIAS BIBLIOGRÁFICAS}

CHANG, I. S.; LE-CLECH, P.; JEFFERSON, B.; JUDD, S. Membrane fouling in membrane bioreactors for wastewater treatment. Journal of Environmental Engineering, v. 128, p. 10181029, 2002.

CHUA, H. C.; ARNOT, T. C.; HOWELL, J. A. Controlling fouling in membrane bioreactors operated with a variable throughput. Desalination, v. 149, p. 225-229, 2002. 
DELGADO, S.; VILLAROEL, R.; GONZÁLEZ, E. Effect on the shear intensity on fouling in submerged membrane bioreactor for wastewater treatment. Journal of Membrane Science, v. 311, p. 173-181, 2008.

LEBEGUE, J.; HERAN, M.; GRASMICK, A. Membrane bioreactor: distribution of critical flux throughout an immersed HF bundle. Desalination, v. 231, p. 245-252, 2008.

LIAO, B. Q.; BAGLEY, D. M.; KRAEMER, H. E.; LEPPARD, G. G.; LISS, S. N. A Review of Biofouling and its Control in Membrane Separation Bioreactors, Water Environmental Research, v. 76, n. 5, p. 425-436, 2004.

MELIN, T.; JEFFERSON, B.; BIXIO, D.; THOEYE, C.; DE WILDE, W.; DE KONING, J.; VAN DER GRAAF, J.; WINTGENS, T. Membrane bioreactor technology for wastewater treatment and reuse. Desalination v. 187, p. 271-282, 2006.

MENG, F.; YANG, F.; SHI, B.; ZHANG, H. A comprehensive study on membrane fouling in submerged membrane bioreactors operated under different aeration intensities. Separation and Purification Technology, v. 59, p. 91-100, 2008.

OLIVEIRA, J. L. G. Medição de vazão de escoamentos bifásicos utilizando tubo de Venturi ou placa de orifício associados a um sensor de fração de vazio com campo elétrico girante. Dissertação de mestrado, UFSC, Florianópolis, SC, Brasil, 2007.

SILVA, M. K. Biorreatores com membranas: uma alternativa para o tratamento de efluentes. Tese de Doutorado, UFRGS, Porto Alegre, RS, Brasil, 2009.

UEDA, T.; HATA, K.; KIKUOKA, Y.; SEINO, O. Effects of aeration on suction pressure in a submerged membrane bioreactor. Water Research, v. 31, p. 489-494, 1997.

WU, J.; HE, C.; ZHANG, Y. Modeling membrane fouling in a submerged membrane bioreactor by considering the role of solid, colloidal and soluble components. Journal of Membrane Science, v. 397, p. 102-111, 2012.

YEO, A.; FANE, A.G. Performance of individual fibers in a submerged hollow fiber bundle. Water Science and Technology, v. 51, p. 165-172, 2005. 